\title{
Pipe-lining Dynamic Programming Processes in Order to Synchronize Energy Production and Consumption
}

\author{
Fariha Bendali \\ LIMOS CNRS/UCA \\ Clermont-Fd, France \\ Email: bendali@isima.fr \\ Jean Mailfert \\ LIMOS CNRS/UCA \\ Clermont-Fd, France \\ Email: mailfert@isima.fr
}

\author{
Alain Quilliot \\ LIMOS CNRS UMR 6158 \\ LABEX IMOBS3, Université \\ Clermont-Auvergne \\ Bat. ISIMA, BP 10125 \\ Campus des Cézaux, \\ 63173 Aubière, France \\ Email: quilliot@isima.fr
}

\author{
Eloise Mole Kamga \\ LIMOS CNRS UMR 6158 \\ 63173 Aubière, France \\ Email: eloise@isima.fr \\ Hélène Toussaint \\ LIMOS CNRS UMR 6158 \\ LABEX IMOB3 \\ Clermont-Fd, France
}

\begin{abstract}
Synchronizing heterogeneous processes remains a difficult issue in Scheduling area. Related ILP models are in trouble. So we propose here a pipe-line collaboration of a dynamic programming process for energy production and consumption scheduling.
\end{abstract}

\section{INTRODUCTION}

$\mathrm{E}$ FFICIENTLY synchronizing heterogeneous process remains a difficult issue when it comes to scheduling. ILP models are flawed by large gaps induced by the relaxation of the integrality constraint (the Big $M$ problem). This difficulty also arises when one wants to plan industrial, domestic or local logistics activities, while relying on local renewable energy production: Due to both market deregulation and emergent technologies, the rise local producers (factories, farms,...) while simultaneously remaining consumers (see $[1,6]$ ) tends to make this issue a trend in Energy Economics. In the context of Labex IMOBS3 project in Clermont-Fd, France, devoted to Innovative Mobility, we are involved into the control of a local micro-plant for hydrogen $\left(\mathrm{H}^{2}\right)$ production, which provides autonomous vehicles in $\mathrm{H}^{2}$ fuel. Researchers rely here on solar power and photolysis $([4,6,7])$ : so the productivity of the process deeply depend on the intensity of solar illumination. Few works address resulting synchronization issue (see general contribution in [1], studies about electric vehicles (Green VRP, PollutionRouting Problem,... in [3, 8]), and industrial processes (see $[2,5])$ under time-dependent energy costs and access restrictions.

Because of the IMOBS3 project, present contribution is about the synchronous management of, on one side, a fleet of small electric vehicles provided with $\mathrm{H}^{2}$ power cells, and, on the other side, a micro-plant in charge of local $\mathrm{H}^{2}$ fuel production. Taken as a whole, resulting model of Section II involves forecasting, safety management and scheduling. We only address here the last issue, while considering only one vehicle, required to perform tasks according to a prefixed order, which periodically goes back to the micro-plant in order to refuel. The micro-plant has its own production/storage restrictions. Relying on ILP is inefficient, and so we first propose in Section III an exact Dynamic Programming Scheme (DPS). But, though this DPS allows us to state a PTAS (Polynomial Time Approximation Scheme) result, it remains time-costly in practice. So we decompose it (Section IV) into 2 DPS sub-processes, one related to the vehicle, and the other one to the micro-plant which collaborate through a pipe-line.

\section{THE ENERGY PRODUCTION/CONSUMPTION PROBLEM (EPC)}

Some vehicle has to perform internal logistics tasks, while following a route $\Gamma$ which starts from some Depot node and ends in the same way after going through stations $j$ $=1, \ldots, M$, according to this order. Start-node Depot has label 0 and End-node Depot has label $M+1$. The time required by the vehicle in order to go from $j$ to $j+1$ is equal to $t_{j}$, (including service time). The vehicle may leave Depot at time 0 and should finish its route no later than some threshold time TMax. It is powered by hydrogen $\left(\mathrm{H}^{2}\right)$ fuel. The capacity of its tank is denoted by $C^{V e h}$ and we know, for any $j=0, \ldots, M$, the $\mathrm{H}^{2}$ amount $e_{j}$ required in order to move from station $j$ to station $j+1$. The initial $\mathrm{H}^{2}$ load of the vehicle is denoted by $E_{0}$, and the vehicle is required to end its trip with at least the same energy load. It comes that the vehicle must periodically refuel. Refueling transactions take place at a micro-plant, close to Depot: The time required by the vehicle in order to move from station $j$ to the micro-plant (from the micro-plant to $j$ ) is denoted by $d_{j}\left(d_{j}^{*}\right)$; by the same way, the energy required in order to move from $j$ to the micro-plant (from the micro-plant to $j$ ) is denoted by $\varepsilon_{j}\left(\varepsilon^{*}\right)$. Figure 1 displays an example of a trip performed by the vehicle along station Depot $=$ 0, 1, 2, 3, 4, 5, 6 = Depot.

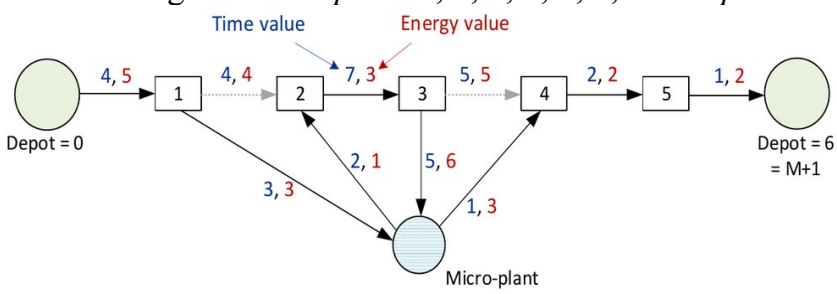

Figure 1. A vehicle trip, with its refueling transactions 
On another side, the micro-plant produces $\mathrm{H}^{2}$ in situ through photolysis\&electrolysis. Resulting $\mathrm{H}^{2}$ is stored inside the micro-plant's tank, with capacity equal to $C^{M P}$. We suppose that the time space $\{0, \ldots, T M a x\}$ is divided into periods $P_{i}=[p . i, p .(i+1)[, i=0, \ldots, N-1$, with TMax $=N . p$ (see Figure 3). We identify index $i$ and period $P_{i}$. If the micro-plant is active at some time during period $i$, then it is active during the whole period $i$, and produces $R_{i}$ hydrogen fuel units. At time 0, the load of the micro-plant tank is $H_{0} \leq$ $C^{M P}$ and the micro-plant is idle. This should also hold at time TMax. Because of safety concerns, the vehicle cannot refuel while the micro-plant is producing and any vehicle refueling transaction takes a whole period $i$. Besides, producing $\mathrm{H}^{2}$ fuel has a cost, which may be decomposed into:

- A constant activation cost Cost $^{F}$, which is charged every time the micro-plant is activated.

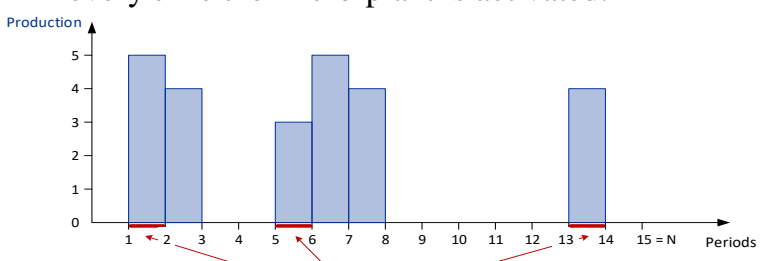

Figure 2. An example of micro-plant activity, with $N=15$

- A time-dependent production cost $\operatorname{Cost}^{V}{ }_{i}$ which reflects the time-indexed prices charged by the electricity provider.

Then the Energy Production/Consumption (EPC) Problem consists in scheduling both the vehicle and the micro-plant in such a way that:

- The vehicle starts from Depot $=0$, visits all stations $j=$ $1, \ldots, M$ and comes back to Depot at some time $T \in[0$, TMax], while refueling every time it is necessary;

- The micro-plant produces and stores in time the $\mathrm{H}^{2}$ fuel needed by the vehicle;

- Both induced $\mathrm{H}^{2}$ production cost Cost and time $T$ are the smallest possible: $\operatorname{Min}=\operatorname{Cost}+\alpha . T$, where $\alpha$ is some scaling coefficient.

Figure 3 below shows the synchronization between the vehicle and the micro-plant of fig. 1,2 , in case $p=2, E_{0}=8$, $H_{0}=4, T M a x=30, \operatorname{Cost}^{F}=7, C^{M P}=15, C^{V e h}=15, \alpha=1$.

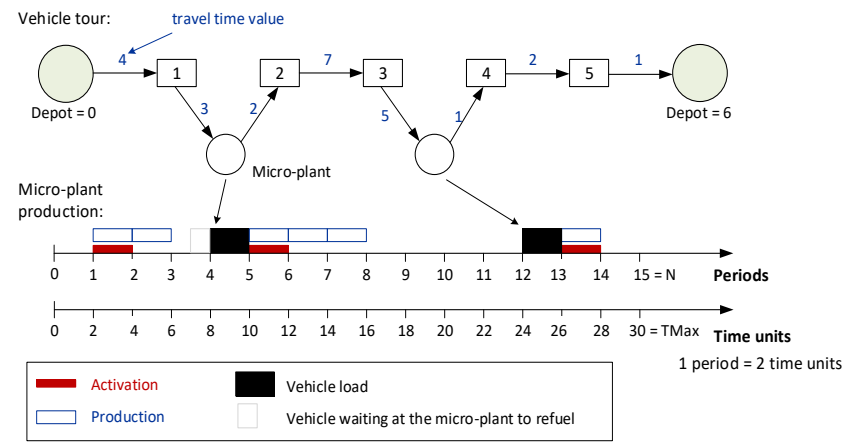

Figure 3. A feasible solution related to Fig. 1 and 2.

\section{A DPS-EPC ALGORITHM.}

EPC is NP-Hard: It can be reduced to Knapsack. We first handle it through DPS (Dynamic Program Scheme):

DPS Time Space and States: The time space is the set $\Delta$ of time pairs $(i, j), i=0, \ldots, N, j=0, \ldots, M+1$.We link periods $i$ and stations $j$ through relations $(<<,>>,==)$ which locate period $i$ with respect to time value $T \in\{0, \ldots, T M a x\}$ :

- $T<<i$ if $T<p . i ; T>i$ if $T \geq p .(i+1)$;

- $T==i$ if $p . i \leq T<p .(i+1)$.

For any such a time pair $(i, j)$, a related state is a 4-uple $s=$ $\left(Z, T, V^{T a n k}, V^{V e h}\right)$, with:

- $Z=1$ : micro-plant active at the end of period $i-1$.

- $V^{T a n k}$ and $V^{V e h}$ are respectively the loads of the micro-plant at the beginning of $i$ and the vehicle when it arrives at $j$;

- $T$ is a value in $0, \ldots$, TMax with the meaning:

○ $T \gg i$ : the vehicle will reach $j$ at time $T$;

○ $T \ll i$ : the vehicle is between $j$ and the microplant, possibly waiting for being refueled;

○ $T==i$ : the vehicle is in $j$, and decides between riding to $j+1$ or to the micro-plant.

Initial state corresponds to time pair $(0,0)$ and 4-uple $s_{0}=$ $\left(0,0, H_{0}, E_{0}\right)$. Final state corresponds to any time pair $(i \leq N$, $M+1)$, and any 4-uple $\left(Z, T \leq \operatorname{TMax}, V^{\text {Tank }} \geq H_{0}, V^{\text {Veh }} \geq E_{0}\right)$.

Decisions/Transitions/Costs. Then a decision $D$ is a 3uple $D=(z, x, \delta)$ in $\{0,1\}^{3}$, with the meaning:

- $z=1 \sim$ the micro-plant produces during period $i$;

- $x$ refers the case $T==i: x=0$ means that the vehicle rides from $j$ to $j+1$ without refueling; $x=1$ means that it refuels at the micro-plant while ridingfrom $j$ to $j+1$.

- $\delta=1 \sim$ the vehicle is located at the micro-plant and decides to refuel during period $i$, forbidding the microplant to be active during this period. It requires $T \ll i$ and $p . i-T \geq d_{j}$.

Decision is taken at the end of period $i-1$. For any time pair $(i, j)$ and state $s=\left(Z, T, V^{\text {Tank }}, V^{V e h}\right)$, no more than 4 decisions $D$ are feasible:

- 1 th case: $T \gg i$. Then the only choice is about $z$.

- 2 th case: $T<i$ and $p . i-T<d_{j}$. The vehicle is moving from $j$ to the micro-plant and cannot refuel yet. Once again, the only choice is about $z$.

- 3 th case: $T \ll i$ and $p . i-T \geq d_{j}$. Then, we have 3 choices: 1$)$. Producing: $z=1 ; \delta=0 ; 2$ ). Refueling: $z=$ $0 ; \delta=1 ; 3)$. Doing nothing: $z=0 ; \delta=0.1$.

- 4 th case: $T==i$. Then we have 4 choices:

$\circ$ Producing and riding towards $j+1: z=1, x=0$. ○ Not Producing, riding towards $\boldsymbol{j}+\mathbf{1}: z=1, x=0$. o Not Producing, riding to micro-plant: $z=0, x=1$. ○ Producing, riding to micro-plant: $z=1, x=1$.

We implement Bellman Equations through a Forward Driven Strategy and denote by DPS-EPC the algorithm designed this way. In order to control th number of states, we need to enhance it with filtering devices. 


\section{A.Filtering through Rounding: A PTAS Result.}

DPS-EPC is in trouble when $M$ and $N$ are large. Still, by considering that 2 states are equivalent when they are equal modulo the $K$ largest bits and extending the notion of state in a well-fitted way, we turn DPS-EPC into an algorithm DPS$\boldsymbol{E P C}(K)$ which allows to state:

Theorem 2 (Polynomial Time Approximation Scheme): For any value $\varepsilon>0$, we may choose $K=K(\varepsilon)$ large enough in such a way that in case EPC admits an optimal solution with value $W^{\text {Opt }}$, then DPS-EPC $(K(\varepsilon))$ yields in polynomial time a solution which is feasible with regards to initial values $(1+\varepsilon / 2) \cdot H_{0}$ and $(1+\varepsilon / 2)$. $E_{0}$, threshold values $(1$ $+\varepsilon) \cdot C^{M P},(1+\varepsilon) \cdot C^{V e h}$ and $(1+\varepsilon)$.TMax and whose cost value is no larger than $W^{o p t}$.

\section{B.Logical Filtering Devices.}

First, we apply the standard Dominance Rule: If, for a given time pair $(i, j)$, state $s_{1}$ dominates state $s_{2},\left(W_{1} \leq W_{2} ; T_{1} \leq T_{2}\right.$ $; Z_{1} \geq Z_{2} ; V^{\text {Tank }}{ }_{1} \geq V^{\text {Tank }} ; V^{\text {Veh }}{ }_{1} \geq \mathrm{V}^{\text {Veh }}{ }_{2}$ ), then we kill $s_{2}$. But this has little filtering power. So, for any time pair $(i, j)$, and related state $s=\left(Z, T, V^{T a n k}, V^{V e h}\right)$, we get rough estimations Fuel and Time of respectively energy and time required in order to allow the vehicle to return from $j$ to Depot, and derive the following logical filtering rules:

1).Makespan Based filtering rule: If (Time $\geq$ TMax $-T$ $+1)$ then kill state $s=\left(Z, T, V^{\text {Tank }}, V^{\text {Veh }}\right)$ related to time pair $(i, j)$, since there is not enough time left for the vehicle to achieve its trip.

2) Energy Based filtering rule: If Fuel $>V^{\text {Veh }} \sum_{k \geq i} R_{k}+$ $V^{\text {Tank }}$ then kill state $s=\left(Z, T, V^{\text {Tank }}, V^{V e h}\right)$ related to time pair $(i, j)$, since there won't be enough energy for the vehicle to achieve its trip.

We go further and pre-compute, for any energy amount $V$, any period number $i$, and any micro-plant $Z$ value, the minimal cost $\operatorname{Cost-Min}(i, V, Z)$ required from the micro-plant to produce $V$ energy units from time $p . i$ on, $Z$ denoting the state of the micro-plant at the end of period $i-1$.Then, for any time pair $(i, j)$ and any state $s=\left(Z, T, V^{T a n k}, V^{V e h}\right)$ with value $W$, we derive a lower bound $L B$ of a best EPC trajectory involving $(i, j)$ and $s$, by setting: $L B((i, j), s)=$ $\alpha$. Time $+\operatorname{Cost-Min}\left(i,\left(\text { Fuel }-V^{\text {Tank }}\right)^{+}, Z\right)+W$. This lower bound allows us to turn $\boldsymbol{D P S}-\boldsymbol{E P C}$ into a greedy procedure $\boldsymbol{G R E E D Y - E P C}$, by keeping, for any time pair $(i, j)$, only the state $s(i, j)$ which minimizes $L B((i, j), s)$. GREEDY-EPC provides us with some feasible value Current-Value and we may apply the following Upper/Lower Bound Based filtering rule 3$)$ : If $L B((i, j), s) \geq$ Current-Value, then kill state $s=\left(Z, T, V^{\text {Tank }}, V^{\text {Veh }}\right)$, related to time pair $(i, j)$.

\section{PIPE-LINE DECOMPOSITION OF DPS-EPC.}

\section{A. The DPS-Vehicle Scheme.}

We do here as if micro-plant were able to provide, at any time, the vehicle with as much as energy it needs. we optimize the Refueling Strategy of the vehicle, that is the $\{0$, 1 ) valued vector $x=\left(x_{j}, j=0 . . M\right)$ and the load vector $L=\left(L_{j}\right.$, $j=0 . . M)$ which tell us at which stations $j$ vehicle will refuel between $j$ and $j+1$, and how much, while minimizing some quantity: $\alpha \cdot T^{\text {End }}+\beta \cdot\left(\Sigma_{j} L_{j} \cdot x_{j}\right)$, where $T^{\text {End }}$ means the ending date of the vehicle trip, and $\beta$ is an auxiliary cost coefficients. We notice that every time the vehicle arrives to the micro-plant, it is sufficient for him to refuel exactly the $\mathrm{H}^{2}$ it needs in order to reach the next refueling transaction. This leads us to the following DPS-Vehicle scheme, whose components time, state and decision come as follows:

- $\quad$ Time Space: the set $J=\{0,1, \ldots, M, M+1\}$.

- State Space: A state $s$ is a 2-uple $s=\left(T, V^{V e h}\right): T$ is the time necessary in order to come back to Depot and $V^{V e h}$ the load at $j$ of the vehicle tank. Its value $W=\alpha . T$ $+\beta . U$, involves the energy amount $U$ which will be wasted by the vehicle before the end of its trip. Initial state (in the sense of a backward driven DPS) is the state $\left(0, E_{0}\right)$ related to $j=(M+1)$. Final states, related to $j=0$, should be any state $\left(\mathrm{T} \leq T M a x, V^{V e h} \leq E_{0}\right)$.

- Decision Space: A decision $x \in\{0,1\}: x=0$ means a no refueling move to $j+1$, and $x=1$ means a refueling move to the micro-plant before reaching $j+1$.

- Backward Driven Strategy: In order to store, for any pair $\left(j, V^{V e h}\right)$, the time and energy amount required in order to achieve tour, we implement Bellman Principle according to a backward driven strategy.

We denote by DPS-Vehicle the resulting DPS algorithm. In order to synchronize it with the $\mathrm{H}^{2}$ Production control, we retrieve from any run a Reduced Refueling Strategy, that is:

- $S=$ number of refueling transactions; Loads $\mu_{s}=$ quantities of $\mathrm{H}^{2}$ which is loaded for every value $s=1 \ldots S$;

- Lower bounds $m_{1}, . . m_{Q}$ and upper bounds $M_{1}, . ., M_{S}$ for the related period numbers $i_{1}, . ., i_{S} \in\{0, . ., N-1\}$, as well as Time Lag coefficients $B_{1}, . ., B_{S}$ which means: For any $s=1 . . S-1, i_{s+1} \geq i_{s}+B_{s}$.

\section{B. The DPS-Prod Scheme}

Let $S, m, M, \mu$ be a Reduced Refueling Strategy, as above. Then we want to schedule the activity of the microplant, that is compute $\{0,1\}$-valued vectors $z$ and $\delta$ with indexation on $i=0 . . N-1$ as in DPS-ECP, in such a way that:

The vehicle may refuel at some periods $i_{1}, . ., i_{\mathrm{S}}$ in a way consistent with time lags and time window constraints induced by the Reduced Refueling Strategy The micro-plant ends with the $\mathrm{H}^{2}$ load as it started; We minimize $\alpha \cdot i_{\mathrm{S}}+\sum_{i=0 . N-1}\left(\operatorname{Cost}^{\mathrm{F}} \cdot y_{\mathrm{i}}+\operatorname{Cost}_{i .}^{V} \cdot z_{i}\right)$. 
We apply a forward driven DPS algorithm DPS-Prod with the following Time, State, and Decision components:

- Time Space: the set $I=\{0 . . N\}$.

- State Space: For any $i=0 . . N$, a state is a 4-uple $E=(Z$, $V^{\text {Tank }}$, Rank, Gap), with Rank in 0..S:

○ $Z=1 \sim$ the micro-plant is active at the end of $i$ - 1 .

- $V^{\text {Tank }}$ is the load of the micro-plant when $i$ starts.

- Rank $\in 1$..S $\sim$ the $\operatorname{Rank}^{\text {th }}$ refueling transaction has been performed and we are waiting for the (Rank + $1)^{\text {th }}$ refueling transaction. Gap means the difference between $i$ and the period when the Rank $k^{\text {th }}$ refueling transaction was performed.

For every $i=0 . . N$, a state $E$ is provided with its current Bellman value $W^{\text {Prod }}$.

○ Initial state is $E^{\text {Start }}=\left(0, H_{0}, 0,0\right)$, with related value $W^{\text {Prod }}=0$, and time value $i=0$;

○ $\quad$ Final states are states $E^{\text {End }}=\left(Z, V^{\text {Tank }} \geq H_{0}, S, 0\right)$, associated with a time value $i \leq N$;

- Decision/Transitions: For any $i=0 . . N, E=\left(Z, V^{T a n k}\right.$, Rank, Gap), a decision is defined as a 2-uple $(z, \delta)$ in $\{0,1\}^{2}$, with the following meaning:

○ $\quad z=1 \sim$ the micro-plant will produce during period $i$;

○ $\delta=1 \sim$ the vehicle will perform its $(\text { Rank }+1)^{\text {th }}$ refueling transaction during period $i$.

Since production and refueling cannot be performed simultaneously, there are only 3 possible decisions:

1). $\underline{z=1, \delta=0} ; 2) . \underline{z=0, \delta=0} ; 3) . \underline{z=0, \delta=1}$.

As for DPS-EPC, we may enhance DPS-Prod through logical and upper/lower bound based filtering devices.

\section{The Pipe-Line Scheme}

Clearly, the simplest way to make above DPS-Vehicle and DPS-Prod interact, is to design the following heuristic Pipe-Vehicle->Production:

\section{Main Steps of Pipe-Vehicle->Production:}

1). Fix $\beta$ and Apply DPS-Vehicle, to the Vehicle instance related to $\alpha, \beta$ : get related Reduced Refueling Strategy;

2). Apply DPS-Prod to resulting Production instance;

3). Reconstruct the whole EPC solution.

Choosing $\beta$ : $\beta$ should reflect the energy production cost. Since we do not know when the refueling transactions take place, we do as if were to be uniformly distributed.

\section{V.NUMERICAL EXPERIMENTS.}

Purpose and Technical Context: We evaluate: 1). the pipe-line decomposition DPS-Vehicle and DPS-Prod; 2). the filtering devices and the greedy procedure described in III.2, while using $\mathrm{C}++$, on Windows 10 with IntelCore i56500@3.20 GHz CPU, 16 Go RAM.

Instances: We fix $N$ and $M$, and randomly generate stations $j$ and Depot and the Micro-Plant as point of the $R^{2}$ space. Then $d_{j}, d^{*}{ }_{j}$ and $t_{j}, e_{j}, \varepsilon_{j}, \varepsilon^{*}{ }_{j}$ respectively corresponds to Euclidean and Manhattan distances. Then we fix $C^{M P}$, $C^{\text {Veh }}, T M A X, \operatorname{Cost}^{F} \geq \operatorname{Inf}_{i} \operatorname{Cost}_{i}^{V}, i=0, \ldots, N-1$.

Outputs: We first run Greedy-EPC with 50 replications, $\Rightarrow$ gap G-Gap to optimality. Next we run DPS-EPC:

1) Only with the Strong Dominance Rule $\Rightarrow S T(1)$ $=$ Maximal number of states for a given pair $(i, j)$,

2) With the 2 Logical Filtering rules $=>S T(2)$;

3) With all filtering rules $=>S T(3)(i, j)$.

Next we run Pipe-Vehicle->Production and get max state/time ST-Veh, ST-Prod, and gap P-Gap to optimality.

\begin{tabular}{|l|l|l|l|l|}
\hline $\begin{array}{l}\text { Instance }(M, \\
N)\end{array}$ & $G-G A P$ & $S T(3)$ & $S T(2)$ & $S T(1)$ \\
\hline $1,(6,27)$ & 18.4 & 11870 & 11369 & 394754 \\
\hline $2,(6,26)$ & 1.5 & 447 & 2619 & 299933 \\
\hline $3,(10,25)$ & 0.0 & 10642 & 25636 & 107228 \\
\hline $4,(10,31)$ & 11.4 & 17526 & 26254 & 310543 \\
\hline $5,(10,46)$ & 0.0 & 21404 & 45014 & 425009 \\
\hline
\end{tabular}

TABLE 1: VALUES $N, M, G$-Gap, $S T(1), S T(2), S T(3)$

\begin{tabular}{|l|l|l|l|}
\hline Inst $(M, N)$ & ST-Veh & ST-Prod & P-Gap \\
\hline $1,(6,27)$ & 22 & 895 & 6.4 \\
\hline $2,(6,26)$ & 18 & 105 & 0 \\
\hline $3,(10,25)$ & 43 & 902 & 0 \\
\hline $4,(10,31)$ & 50 & 1088 & 2.9 \\
\hline $5,(10,46)$ & 52 & 1385 & 0 \\
\hline
\end{tabular}

TABLE 2: VALUES $N, M$, ST-Veh, ST-Prod, P-Gap

Comment: Dominance rule has little impact, logical anticipation and optimistic estimation rules are significantly more efficient, while the pipe-line scheme DPS-Vehicle -> DPS-Production offers a good tradeoff time/accuracy.

\section{CONCLUSION}

In the future, we shall deal with uncertainties, address the vehicle route issue, manage the on line context.

\section{REFERENCES}

[1]. S.Albers: Energy-efficient algorithms; Communications of ACM 53, 4, p 86-96, (2010). https://dl.acm.org/doi/10.1145/1735223.1735245

[2]. ARTIGUES, E.HEBRARD, A.QUILLIOT, H.TOUSSAINT: Models and algorithms for evacuation problems; IEEE Proc. FEDCIS WCO12, Leipzig, 4 pages, (2019). https://doi.org/10.15439/2019F90

[3]. L.Benini, , A.Bogliolo, G.De Micheli: A survey of design techniques for system level dynamic power management; IEEE Transactions of Very Large Scale Integratio Systems, 8, 3, p 299-316, (2000). https://dl.acm.org/doi/10.1145/1403375.1403402

[4]. C.C.Chan. The state of the art of fuel cell vehicles. Proc. of the IEEE, 95, p 704-718, (2007). DOI: 10.1109/JPROC.2007.892489

[5]. P.Chretienne, A.Quilliot: A polynomial algorithm for the non idling scheduling problem; DAM, 20 pages, (2018). https://doi.org/10.1016/j.dam.2013.01.019

[6]. C.Grimes, O.Varghese, S.Ranjan. Light, water, hydrogen: photoelectrolysis. Springer US, (2008). ISBN 978-0-387-33198-0

[7]. S.Licht. Thermochemical and Thermal/Photo Hybrid Solar Water Splitting, Springer New York, NY, (2008). https://link.springer.com/chapter/10.1007/978-0-387-72810-0_5

[8]. C. Lin, K.L.Choy, G.T.Ho, S.H. Chung, H.Lam. Survey of green vehicle routing problem. Expert Systems Applications, 41, p 11181138, (2014). https://doi.org/10.1016/j.eswa.2013.07.107. 